\title{
Biomolecule Encapsulated Carbon Nanotubes Using Nano Processing in Electrolyte Plasmas
}

\author{
Toshiro Kaneko and Rikizo Hatakeyama \\ Department of Electronic Engineering, Tohoku University, \\ 6-6-05, Aoba, Aramaki, Aoba-ku, Sendai 980-8579, Japan. \\ Tel: +81-22-795-7046, Fax: +81-22-263-9375 \\ e-mail: kaneko@ecei.tohoku.ac.jp
}

\begin{abstract}
An encapsulation of biomolecules such as DNA and ionic liquids into single-walled carbon nanotubes (SWNTs) is demonstrated using an ion irradiation method in electrolyte plasmas. The DNA negative ion irradiation can be controlled by varying electric fields and irradiation time. Based on the Raman spectroscopy analysis, DNA is confirmed to be inserted into SWNTs. Furthermore, the positive and negative ions of the ionic liquids are also encapsulated into SWNTs and modify the electronic state of SWNTs depending on the polarity of the ions. Key words: electrolyte plasmas, DNA, ionic liquids, carbon nanotubes,
\end{abstract}

\section{INTRODUCTION}

Single-walled carbon nanotubes (SWNTs) [1] are one of the most interesting and promising materials because they can widely be applied for mechanical, electronic, optical, and medical fields. In particular, electronic applications such as nano electronic devices and chemi$\mathrm{cal} / \mathrm{bio}$ sensors have great potentials to be realized in the near future. Although SWNT-based field effect transistors show marvelous electron transport properties, they only show a p-type characteristic [2]. Therefore investigations related to the electronic modification of SWNTs have been desired.

In this sense, a biological molecule such as DNA is one of the candidates for the electronic modification, because DNA is well known to consist of four kinds of base, each of which has a different electronic property [3], and the base sequence has come to be easily controlled recently. Therefore, the control of electronic properties of SWNTs can be performed by encapsulating DNA, the base sequence of which is properly selected. Since DNA is a typical polyelectrolyte due to its phosphoric acid part which has negative charge in solution, we can regard a DNA solution as an electrolyte plasma [4]. The behavior of the ions in the electrolyte plasmas is basically understood by Debye and Huckel's theory [5], the scheme of which is almost the same as that in the gas phase plasmas.

On the other hand, ionic liquids [6,7] have recently attracted much interest because of their unique properties, such as existence of only positive and negative ions, nonvolatile, and so on. Since the ionic liquids have high polarity, the encapsulation of the ionic liquids is also expected to modify the electronic characteristics of SWNTs.

In this paper, it is demonstrated that the formation of new functional SWNTs encapsulating the biomolecule such as DNA and the ionic liquids by nano processing in the electrolyte plasma using the principle of electrophoresis and dielectrophoresis $[8,9]$.

\section{EXPERIMENTAL APPARATUS}

In this experiment, "the plasma ion irradiation method" [10] which is conventionally used in gas plasmas is applied to the electrolyte plasmas in order to encapsulate biomolecules into SWNTs because the behavior of the ions in the electrolyte plasmas is expected to be the same as that in the gas plasmas. The schematic of an experimental apparatus for DNA irradiation is shown in Fig. 1. A direct-current (DC) electric field is applied to the DNA electrolyte plasma (DNA solution) by applying the DC bias voltage $\left(V_{\mathrm{DC}}=0-20 \mathrm{~V}\right)$ to micro gap electrodes $(d=1 \mathrm{~mm})$ in order that the DNA negative

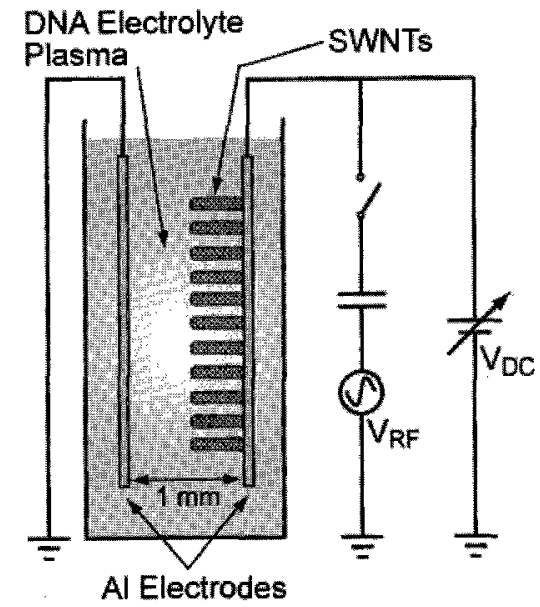

Fig. 1 Schematic of an experimental apparatus for the formation of DNA encapsulated single-walled carbon nanotubes. 
ions are moved to the anode electrode coated with SWNTs in the same way as electrophoresis, where SWNTs are treated by heat for opening its tips in advance [11]. On the other hand, a radio-frequency (RF) electric field in the electrolyte plasma, which is generated by the applied RF voltage $\left(V_{\mathrm{RF}}=0-150 \mathrm{~V}\right)$, plays a role in stretching random-coil-shaped DNA molecules due to the interaction between induced dipoles in the DNA molecules and the external RF electric field (dielectrophoresis) [12].

In this experiment, several kinds of single-stranded DNA which have different bases and length are used. The bases of adenine, thymine, guanine, and cytosine are represented by $\mathrm{A}, \mathrm{T}, \mathrm{G}$, and $\mathrm{C}$, respectively. In addition, the number of the bases is denoted by subscript, for example, the DNA molecule which consists of fifteen adenines is represented by $A_{15}$.

\section{EXPERIMENTAL RESULTS}

\subsection{DNA irradiation}

The DNA negative ion irradiation with the DC electric field is investigated using UV absorption spectra of the DNA solution, where we can find a specific absorption peak around $260 \mathrm{~nm}$ originating from the DNA molecules. In this experiment, only DC electric field is applied. With an increase in irradiation time $(t)$, the $a b$ sorbance is found to decrease. This phenomenon is one of the evidence of the ion irradiation. The concentration of the solution $(c)$ is obtained using the normalization that the value of unity in absorbance corresponds to about $c=35 \mu \mathrm{g} / \mathrm{ml}$. The time evolution of the concentration of the DNA solution with $V_{\mathrm{DC}}$ as a parameter is plotted in Fig. 2. It is found the concentration decreases with the irradiation time and an increase in $V_{\mathrm{DC}}$. The results indicate that the DNA irradiation can be controlled by changing the strength of DC electric field and irradiation time. In the case of $V_{\mathrm{DC}}=10 \mathrm{~V}$, almost all of the DNA molecules appear to be irradiated to the anode after $10 \mathrm{~min}$. Based on these results, the irradiation of DNA to SWNTs is performed within 10 minutes for $V_{\mathrm{DC}}$ $=10 \mathrm{~V}$. The RF electric field does not affect the motion

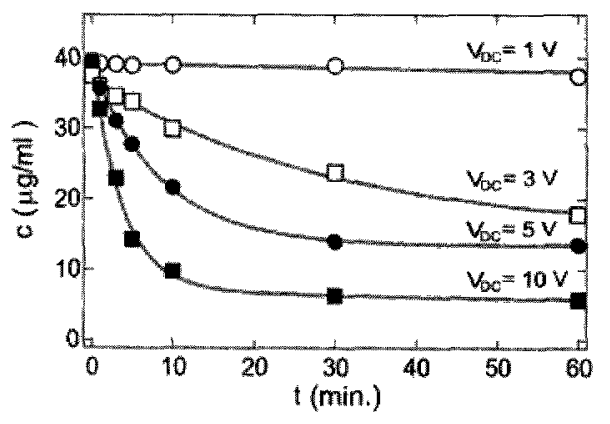

Fig. 2 Time evolution of the concentration of DNA solution with $V_{\mathrm{DC}}$ as a parameter.

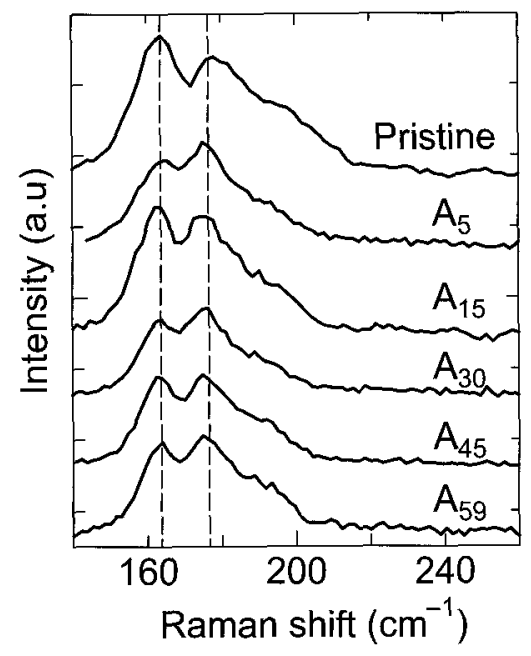

Fig. 3 Raman spectra of SWNTs in the RBM region after DNA irradiation with the number of bases as a parameter. The excitation wavelength of the laser is $488 \mathrm{~nm} . V_{\mathrm{DC}}=10 \mathrm{~V}$ and $V_{\mathrm{RF}}=0 \mathrm{~V}$.

of the DNA molecules but the conformation can be changed under our condition. After applying the electric fields, SWNTs are analyzed by Raman scattering spectroscopy.

Figure 3 presents Raman spectra of SWNTs in the region of radial breathing mode (RBM). Since RBM mode is the stretching mode of SWNTs in the radial direction, this mode is considered to be sensitive to the encapsulation of other materials in SWNTs. The spectrum shape is found to change in any case after the DNA negative ion irradiation compared with that of pristine SWNTs, which is especially reflected in the drastic decrease of the peak intensity at $164 \mathrm{~cm}^{-1}$. The changes of the peak intensity in the range of RBM are considered to be the one of the

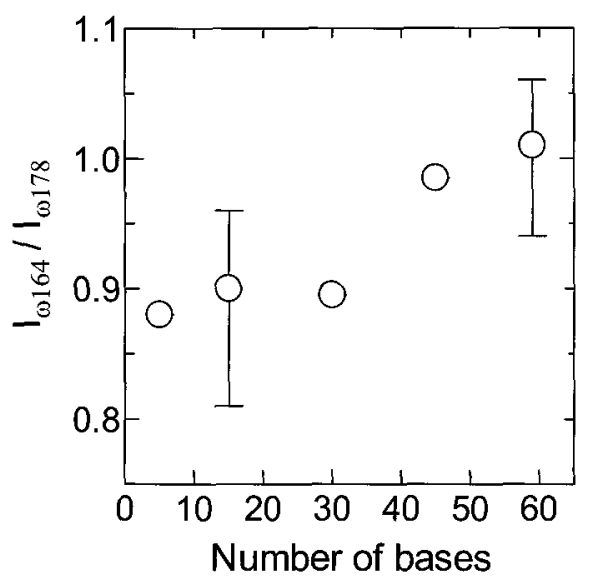

Fig. 4 The ratio of the peak intensity at $164 \mathrm{~cm}^{-1}$ to that at $178 \mathrm{~cm}^{-1}$ in Raman spectra as a function of the number of bases, which is obtained from Fig. 3. A smaller value of the peak intensity ratio denotes a higher yield of the DNA encapsulation. 
phenomena associated with interaction between encapsulated DNA and SWNTs. Although the effect of interaction may be very small, the signal of RBM is considered to be very sensitive to the condition of the SWNT inner region. As a result, even a slight effect of interaction has the possibility for changing RBM spectra, giving indirect evidence of the encapsulation of other materials inside SWNTs. In the case of $\mathrm{A}_{5}$ used, the reduction of the peak intensity at $164 \mathrm{~cm}^{-1}$ is most significant compared with the other longer DNA molecules used, which means that shorter DNA is more easily encapsulated.

The intensity ratio of characteristic two peaks at 164 $\mathrm{cm}^{-1}$ and $178 \mathrm{~cm}^{-1}$ in Fig. 3 is plotted as a function of the number of bases, as shown in Fig. 4. The ratio of the two peaks $I_{\omega 164} / I_{\omega 178}$ decreases with a decrease in the number of bases. As mentioned above, the encapsulation efficiency is considered to be enhanced when the number of bases is decreased because the conformation of DNA is not stretched but random-coiled under the present condition without $V_{\mathrm{RF}}$. Therefore, the ratio indicates the yield of the encapsulation of the DNA molecules in SWNTs and a smaller value of $I_{\omega 164} / I_{0178}$ corresponds to a higher encapsulation yield. Accordingly, when only the DC electric filed is applied in order to encapsulate the DNA molecules, long-length DNA molecules are difficult to be encapsulated into SWNTs spontaneously.

Effects of the sheath electric fields in front of the electrodes are investigated by changing the concentration of the DNA solution because the Debye length, i.e., the characteristic length of the sheath electric fields can be controlled by the concentration which corresponds to the ion density in the electrolyte plasma. Raman spectra of the SWNTs after DNA ion irradiation are measured as a function of the concentration of the DNA solution. The spectrum shape is found to gradually change with an increase in the concentration, which is reflected in the

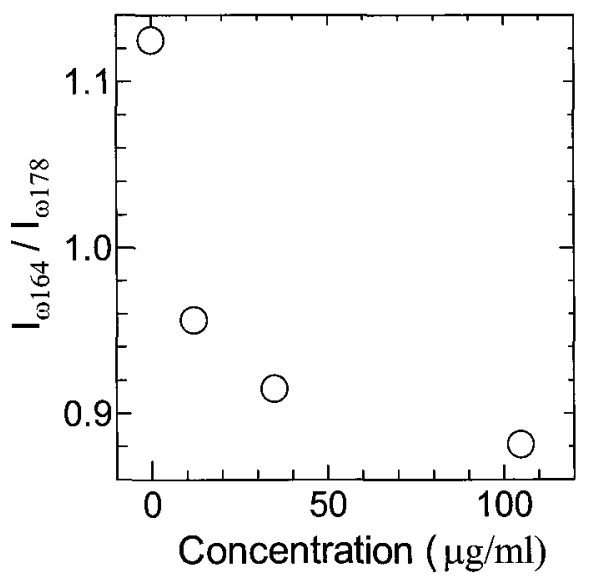

Fig. 5 The ratio of peak intensity at $164 \mathrm{~cm}^{-1}$ to that at $178 \mathrm{~cm}^{-1}$ in Raman spectra of the SWNTs as a function of the concentration of the DNA solution. $\mathrm{A}_{5}, V_{\mathrm{DC}}=10 \mathrm{~V}, V_{\mathrm{RF}}=0 \mathrm{~V}$.

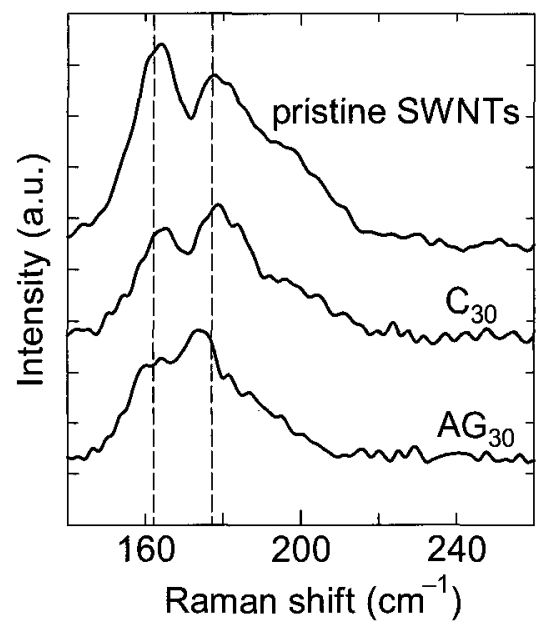

Fig. 6 Raman spectra of SWNTs after DNA irradiation with the base sequence as a parameter. The excitation wavelength of the laser is $488 \mathrm{~nm} . V_{\mathrm{DC}}=10 \mathrm{~V}$ and $V_{\mathrm{RF}}=150 \mathrm{~V}$.

decrease in the ratio of peak intensity at $164 \mathrm{~cm}^{-1}$ to that at $178 \mathrm{~cm}^{-1}$ as presented in Fig. 5. In the case of high concentration, the number of DNA which is irradiated to SWNTs increases, and furthermore, the sheath electric field is expected to become large due to the shortened Debye length. As a result, DNA is more easily encapsulated. Therefore, the change of the Raman spectra is considered to be enhanced by the interaction between the encapsulated DNA and the SWNTs.

In order to investigate effects of a base sequence on the encapsulation, another base such as cytosine and guanine included in DNA is used for the same procedures as shown in Fig. 6, where $V_{\mathrm{DC}}=10 \mathrm{~V}$ and $V_{\mathrm{RF}}=150$ $\mathrm{V}$. In this situation, the Raman spectrum of $\mathrm{AG}_{30}$ is drastically changed compared with that of $\mathrm{C}_{30}$. Figure 7 shows redox potentials (reduction and oxidation potentials) of the four kinds of bases, where guanine and cytosine have the lowest and highest oxidation potentials,

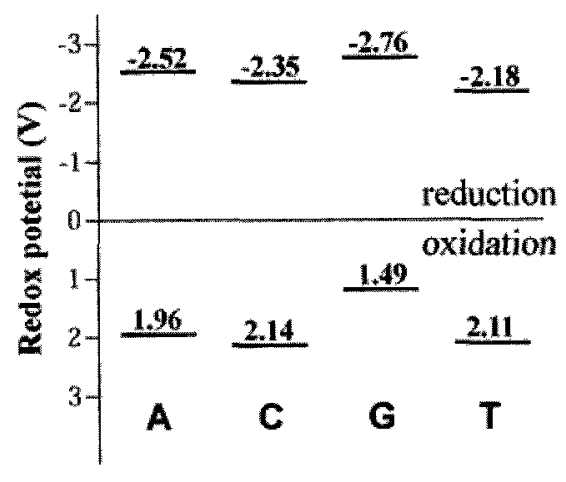

Fig. 7 Redox potentials of the four kinds of bases. Guanine and cytosine have the lowest and highest oxidation potentials, respectively. 
respectively. Therefore, the change in the Raman spectrum of $\mathrm{AG}_{30}$ encapsulated SWNTs is caused by the lowest oxidation potential (electron-donor-like property) of guanine, while the highest oxidation potential of cytosine gives a small effect to SWNTs. This means that the electronic modification of the SWNTs can be controlled by changing the base sequence of encapsulated DNA.

\subsection{Ionic liquid irradiation}

Since DNA is dissolved in water in this experiment, the SWNTs contains not only DNA but also water molecules, resulting in low encapsulation yield of DNA. If the electrolyte such as DNA exists without solvent, high encapsulation yield is expected to be realized. In this sense, we introduce ionic liquids as a new kind of liquid. The ionic liquids have the greatly interesting characteristics such as their composition consisting of only positive and negative ions without neutral solvent, extremely low vapor pressure, high heat capacity, and nonflammable. Furthermore, recently, the ionic liquids can be synthesized by the bases of DNA [13]. Therefore, the ionic liquid is the most suitable liquid for the formation of DNA encapsulated SWNTs without commingling of solvent. Here, in the beginning, we use the popular ionic liquids consisting of imidazolium salts, instead of the DNA ionic liquid.

Figure 8 shows a schematic of an experimental apparatus for the formation of the ionic liquids encapsulated carbon nanotubes. In this case, the SWNTs are coated on both the anode and cathode electrodes for investigating the effects of positive and negative ions of the ionic liquids on the electronic properties of SWNTs. The ionic liquid used in this experiment is 1-buthl-3-methyl imidazolium tetrafluoroborate $\left(\left[\mathrm{C}_{8} \mathrm{H}_{15} \mathrm{~N}_{2}\right]^{+}\left[\mathrm{BF}_{4}\right]^{-}\right)$. The distance between the electrodes is $1 \mathrm{~mm}$, which is the same as the experiments on the DNA irradiation.

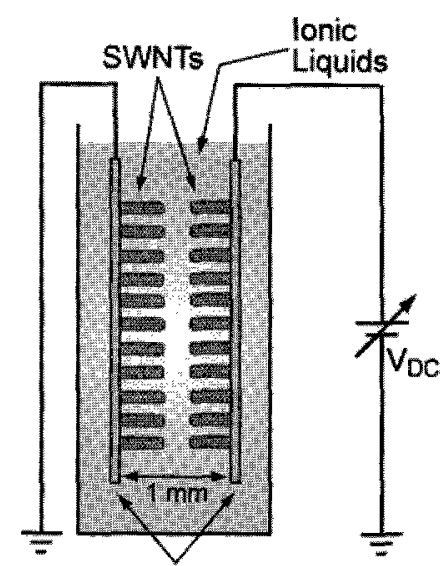

Al Electrodes

Fig. 8 Schematic of an experimental apparatus for the formation of ionic-liquids encapsulated carbon nanotubes.

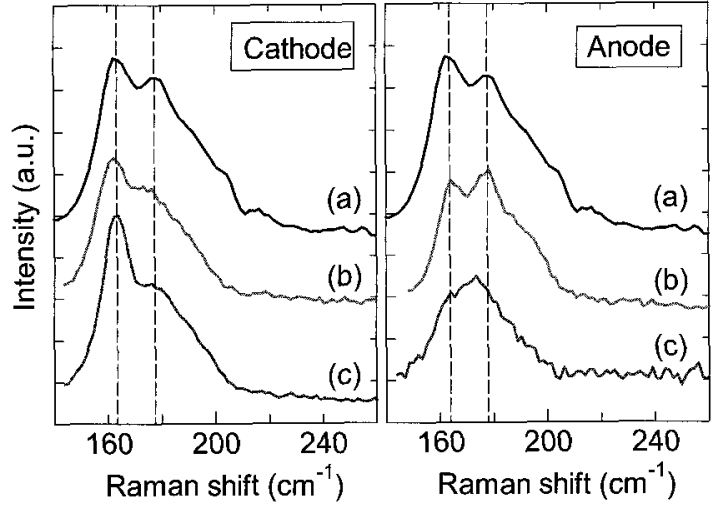

Fig. 9 Raman spectra of SWNTs after ionic liquid irradiation. $V_{\mathrm{DC}}=5 \mathrm{~V}$ and $V_{\mathrm{RF}}=0 \mathrm{~V}$. (a) Pristine, (b) $t=5 \mathrm{~min}$, (c) $t=10 \mathrm{~min}$.

Figure 9 shows Raman spectra of SWNTs coated on the anode and cathode electrodes as a function of ionic-liquid irradiation time for $V_{\mathrm{DC}}=5 \mathrm{~V}$ and $V_{\mathrm{RF}}=0 \mathrm{~V}$. The ratio of peak intensity at $164 \mathrm{~cm}^{-1}$ to that at 178 $\mathrm{cm}^{-1}$ in the Raman spectra in both the anode and cathode cases is clearly changed compared with the case of the pristine SWNTs. Furthermore the peak intensity ratio is found to depend on the polarity of the electrode. Owing to the encapsulation of the positive or negative ions of the ionic liquid, electronic state of the SWNTs is considered to be greatly varied by charge transfer between the SWNTs and the ionic liquid.

By using this electronically modified ionic liquid encapsulated SWNTs, we fabricate the SWNT based FET device. These SWNTs samples are ultrasonically dispersed in $N, N$-dimethylformamide first and then spincoated on FET substrates, each of which consists of $\mathrm{Au}$ drain-source electrodes on a $\mathrm{SiO}_{2}$ insulating layer. $\mathrm{A}$ heavily doped $\mathrm{Si}$ substrate serves as a backgate. The detailed fabrication process for FET devices can be found elsewhere $[14,15]$. The electronic transport measurements, i.e., the drain-source current $I_{\mathrm{DS}}$ as a function of the gate voltage $V_{\mathrm{G}}$ for fixed drain-source voltage $V_{\mathrm{DS}}$, are performed at room temperature under vacuum conditions on a semiconductor parameter analyzer (Agilent $4155 \mathrm{C}$ ). The transport property of pristine semiconducting SWNTs is well known to exhibit the p-type behavior as shown in Fig. 10(a), where a characteristic curve of $I_{D S}-V_{G}$ is described for $V_{D S}=1 \mathrm{~V}$. Figure $10(b)$ presents the transport property of the ionic-liquid irradiated SWNTs on the anode, where the negative ions of the ionic liquid are irradiated to the SWNTs. The typical p-type characteristic is observed, but the threshold voltage $\left(V_{\mathrm{th}}\right)$ for hole conductance is found to shift from -20 $\mathrm{V}$ to $+4 \mathrm{~V}$ compared with that of pristine SWNTs, indicating that the p-type behavior of the SWNTs is enhanced by the encapsulation of the negative-ion of the ionic liquid. In contrast, the transport property of the ionic-liquid irradiated SWNTs on the cathode drastically 
(a)

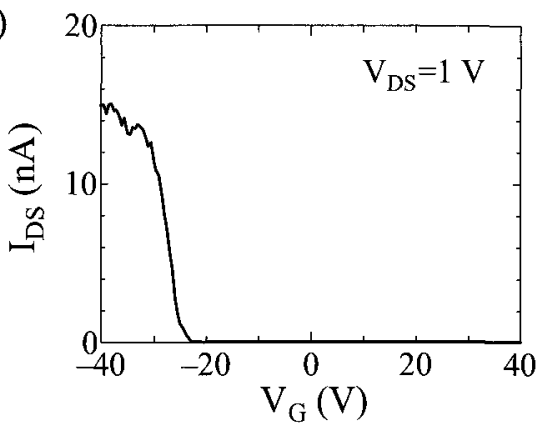

(b)

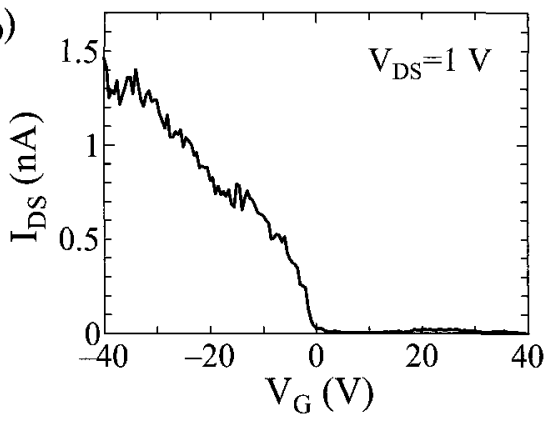

(c)

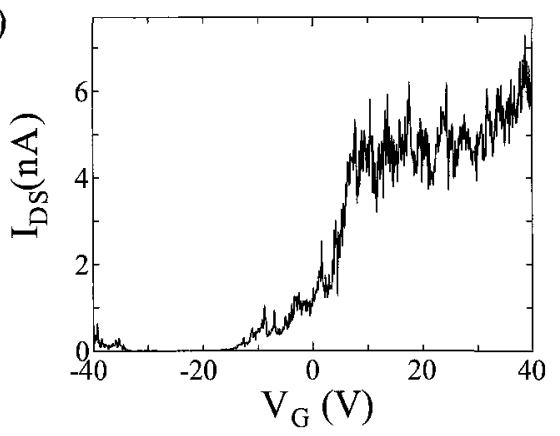

Fig. 10 Source-drain current ( $\mathrm{I}_{\mathrm{DS}}$ ) vs gate voltage $\left(V_{\mathrm{G}}\right)$ characteristics at room temperature for (a) pristine SWNT, (b) negative-ion irradiated SWNT, and (c) positive-ion irradiated SWNT.

changes to an n-type semiconductor [Fig. 10(c)]. This n-type characteristic is attributed to the charge transfer between the positive ions of the ionic liquid and local parts of SWNTs.

Based on these results, encapsulation of the positive or negative ions of the ionic liquid cause the modification of electronic characteristics of the SWNT-based FET. Therefore, the formation of $p$-n junction in SWNTs could be realized by encapsulating ionic liquids as both the electron donor and acceptor.

\section{CONCLUSION}

The DNA encapsulated SWNTs are created by applying the electric fields to the substrate coated with open-ended SWNTs, which is immersed in the electrolyte plasma. Based on the Raman spectroscopy analysis, the encapsulation of DNA inside SWNTs is enhanced when the base length of DNA molecules decreases. Furthermore, the Raman spectrum of SWNTs encapsulating
DNA with guanine is drastically changed compared with that encapsulating DNA which consists of only cytosine. Therefore, the DNA encapsulated SWNTs can possibly be used as novel modified-SWNTs by changing the sequence of DNA.

On the other hand, it is found that the ionic liquids which have high polarity can be encapsulated into SWNTs and the negative and positive ions of the ionic liquids have the different effect on the electronic property of SWNTs.

\section{ACKNOWLEDGMENT}

This research was partly carried out at the Laboratory for Nanoelectronics and Spintronics, Research Institute of Electrical Communication, Tohoku University. Part of this work was carried out under the Cooperative Research Project Program of the Research Institute of Electrical Communication, Tohoku University. This work was also supported by a Grant-in-Aid for Scientific Research from the Ministry of Education, Culture, Sports, Science and Technology, Japan.

\section{REFERENCES}

[1] S. Iijima and T. Ichihashi, Nature 363, 603 (1993).

[2] J. W. G. Wildoer, L. C. Venenma, A. G. Rinzler, R. E. Smalley, and C. Dekker, Nature 391, 59 (1998).

[3] K. -H. Yoo, D. H. Ha, J. -O. Lee, J. W. Park, J. Kim, J. J. Kim, H. -Y. Lee, T. Kawai, and H. -Y. Choi, Phys. Rev. Lett. 87, 198102 (2001).

[4] T. Kaneko, T. Okada, and R. Hatakeyama, Contrib. Plasma Phys. 47, 57 (2007).

[5] P. Debye and E. Huckel, Physkalische Zeitschrift, 24, 185 (1923).

[6] T. Welton, Chem. Rev. 99, 2071 (1999).

[7] K. R. Seddon, Nature Mater. 2, 363 (2003).

[8] T. Okada, T. Kaneko, R. Hatakeyama, and K. Tohji, Chem. Phys. Lett. 417, 288 (2006).

[9] T. Okada, T. Kaneko, and R. Hatakeyama, Jpn. J Appl. Phys. 45, 8335 (2006).

[10] R. Hatakeyama, T. Hirata, and G. -H. Jeong, Plasma Source Sci. Technol. 13, 108 (2004).

[11] S. Bandow, M. Takizawa, H. Hirahara, M. Yudasaka, and S. Iijima, Chem. Phys. Lett. 337, 48 (2001).

[12] M. Washizu and O. Kurosawa, IEEE Trans. Ind. Appl. 26, 1165 (1990).

[13] A. M. Leone, S. C. Weatherly, M. E. Williams, H. H Thorp, and R. W. Murray, J. Am. Chem. Soc. 123, 218 (2001).

[14] T. Izumida, R. Hatakeyama, Y. Neo, H. Mimura, K. Omote, and Y. Kasama, Appl. Phys. Lett. 89, 093121 (2006).

[15] Y. Li, R. Hatakeyama, T. Kaneko, T. Izumida, T. Okada, and T. Kato, Appl. Phys. Lett. 89, 093110 (2006).

(Recieved December 25, 2007 ; Accepted May 28, 2008) 